\title{
Vertical Handover based Context Awareness Concept for Ubiquitous Access
}

\author{
Afif Mériem, \\ Mediatron : Multimedia Mobile Radio Networks Research Unit \\ Higher School of communication of Tunis (SupCom), \\ Carthage University Tunis, Tunisia
}

\begin{abstract}
With the advent of the third and fourth generation, mobile radio networks are entering a new phase of their evolution. These systems must allow mobile users to access their personalized services and their environment regardless of the network radio access technology and the used terminal type. Current solutions are not sensitive to the context and do not take into account parameters that change with the dynamic component constitution of the mobile environment. In this paper we are interested to the adaptation of services to networks with different radio access technologies while maintaining the requested quality of service and the mobile user context. Simulation results show that the effects of the use of context awareness in the decision of handover can quickly converge to a fixed optimal choice while guaranteeing the free movement of mobile hosts across different networks depending on the required QoS.
\end{abstract}

\section{General Terms}

Mobility Management, Ubiquitous access, Wireless Networks

\section{Keywords}

Context Awareness, Vertical Handover, QoS, mobility, heterogeneity

\section{INTRODUCTION}

The user is surrounded by an intelligent environment within which intelligent devices are in a completely transparent evolution. This ever-changing environment affects with the same way different user behaviors and the provided applications. This evolution depends on many elements called "context." The Context has a crucial role in mobile networks and information technology and its use in service discovery helps to refine and adapt the dynamic research for radio interfaces. In other terms, one of the main features of future communication systems is nomadism: the desirable characteristics of nomadism include independence of location, information platform, communication devices, and an optimal throughput.

To take advantage of this heterogeneity, "context awareness" should be considered at different levels of the communication stack. It is defined as a design model for applications where the application uses knowledge related to a set of environment states to determine and modify the behavior of the offered services. Context management allows the network provided application to optimize its behavior according to the context in which it is located. The context awareness takes into account the dynamic changes of the environment in term of provided network technologies to achieve a fixed and optimal choice. To exploit the concept of context awareness, the traditional handover decisions are based primarily on the signal strength which is not enough. To choose the optimal point of attachment between the multitude of available ones in the network, there is a need for a more intelligent handover decision mechanism. In the next network generations of heterogeneous mobile radio systems, one of the major challenges is the vertical handover, the switching of the mobile from one serving cell to other offering different radio access technologies. Indeed, users can freely switch from one network to another. But what we seek through this study is to converge quickly to an optimal choice while ensuring the free movement of mobile users across different networks.

The concept of handover based context aware can be defined as a procedure that selects a target point of attachment for a handover execution, based not only on the signal quality, but also on knowledge of the mobile node and the provided context information of the network to take an intelligent and optimized decision. Thus, a new point of attachment must be chosen supporting optimally user applications.

The reminder of this paper is organized as follows. In section 2 , we define the concept of context awareness. In section 3 , the context management strategy from different point of view is resumed. Then, we present related works which develop vertical handover decision based context awareness, in next section. In section 5, we describe the proposed algorithm which combines static and dynamic context exchange approaches. In the next section, simulation results are presented and performance analysis is depicted in section 7 . Finally, we conclude our present work and we give a short idea for our future research.

\section{CONTEXT AWARNESS}

\subsection{How to Define a Context?}

Define exactly the term "context" was the focus of many research groups. Different definitions have been proposed to describe the term. According to Schils [1], who invented the term "Context Aware Computing," the context is defined in relation with the location, identity of the object and the surrounding people. Brown [1] adds to the definition of Schils the notion of time, season, temperature, etc. Dey [1] is the 
first who gave us a precise definition of context : "Context is any information that can be used to characterize the situation of an entity. An entity is a person, place, or object that is related to the interaction between a user and the application including the user and application themselves. "

\subsection{Context Awareness IT Point of View}

The context awareness [2] is defined by the ability to align the service with its environment. Indeed, it is to adapt applications to new contexts such as the ability of the equipment. In fact, a system service, as shown in Figure 1, allows for accommodating services such as voice, video, data downloading to various computer equipment such as phone, PDA, laptop PC based on the characteristics of these devices such as their abilities. This adaptation takes into account the environment in which the user is located, it comes just at the time of connection establishment. The context awareness is to integrate the context in communications for local scope. This approach allows the application to optimize its behavior depending on the context in which it is located.

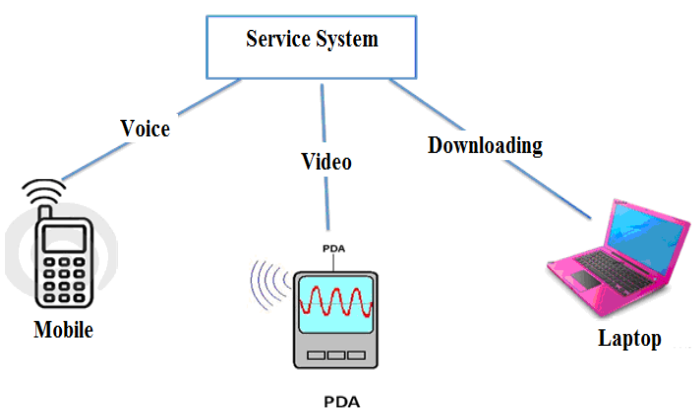

Figure 1. Context Awareness according to IT.

\subsection{Context Awareness Mobility Point of View}

Mobility viewpoint to the context awareness is interested to wide area networks that are not necessarily derived from the same domain of application. Here the context occurs at any time during the handover of a given communication and also during the communication. Indeed, it is to adapt mobile equipment required services, as described in Figure 2, to the various systems using different access technologies, providing both a good quality of service and an optimal routing throughout the mobile active session. For example, a mobile user who is in an area covered by the WiMax network, which uses voice, data and video, and knowing that these services have different physical characteristics in terms of throughput and bandwidth, can attach to other cells WLAN and GPRS to ensure a better quality of service and may be lower cost.

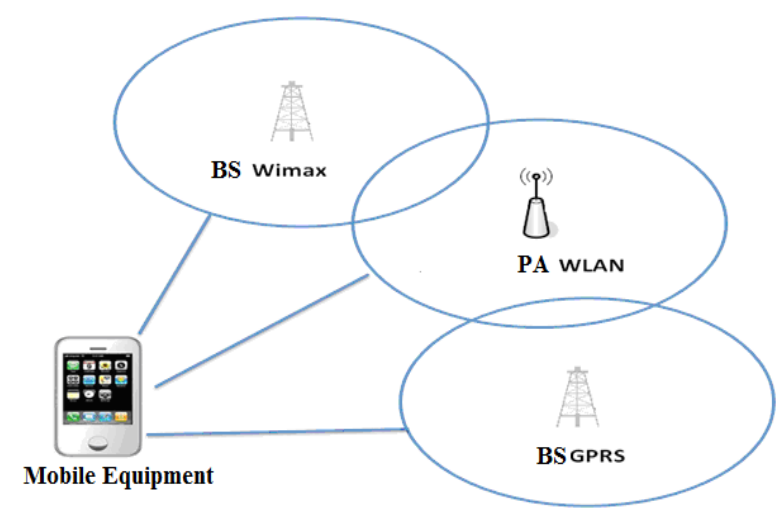

Figure 2. Context Awareness according to mobility aspect.

We can therefore say that a context-aware system must be able to perceive the situation of the user according to its environment and adapt the system behavior to the suggested situation in term of required services depending on their quality.

\section{CONTEXT MANAGEMENT}

\subsection{The problems Associated With Context Information}

The context-awareness is all type of information presents in the environment which describes the situation of the user required service and the network in terms of location, time, user activity and network provider [3-4]. It can be got back from a big variety of sources and may be formed by various metrics such as network availability, network capability, bandwidth, signal strength, user preference, access cost, quality of service, security, mobility location, user activity, etc. Effective use of context information is not simple due to three main reasons:

- The context information can be distributed among the different network elements, and it's hard to find all the background information in a single network entity. For example, information about the user profile may be available in mobile devices, in user home networks or in the network of some service providers.

- The context information is dynamic and can change frequently and thus the accuracy is lost in time.

- Since the context information change over time, the algorithms for interpreting context information must be updated frequently.

In most cases, applications use only a small fraction of background information available for specific services. In particular cases of mobile networks, there are other problems such as instability and limited radio resources, limited power and mobility management during roaming situations. 


\subsection{Context Management for Radiomodile Network}

Context management, dynamic or distributed [5], is not only executed at application layer, but also at network layer and data link layer, while clearly separating the application specific context to the general context. Figure I. 3 shows that this separation allows the creation of generic mechanisms for all types of applications that enable the collection of context information. The purpose of context management is to facilitate the collection, pretreatment, distribution and use of contextual information.The process of gathering contextual information is implemented independently of the type of application. While the distribution and use of this information is for a specific applications. What makes the process of collecting contextual information independent of the type of application is its ability to collect this information from the context supplier using a generic format. The generic information collected from a context collection point is shared between different applications. However, each application has its own requirements, which is why the generic context information must be translated into a format that every application can analyze it and the distribution of this information must be made by means of a protocol adapted to each application. Context adapters are specific application adapters that allow the selection of information collected and the adjustment of such information with each request. Note that different adapters can interact with the same context collection point as shown in Figure 3. Each adapter sends the appropriate information to a context client adapter which interacts with a specific client environment

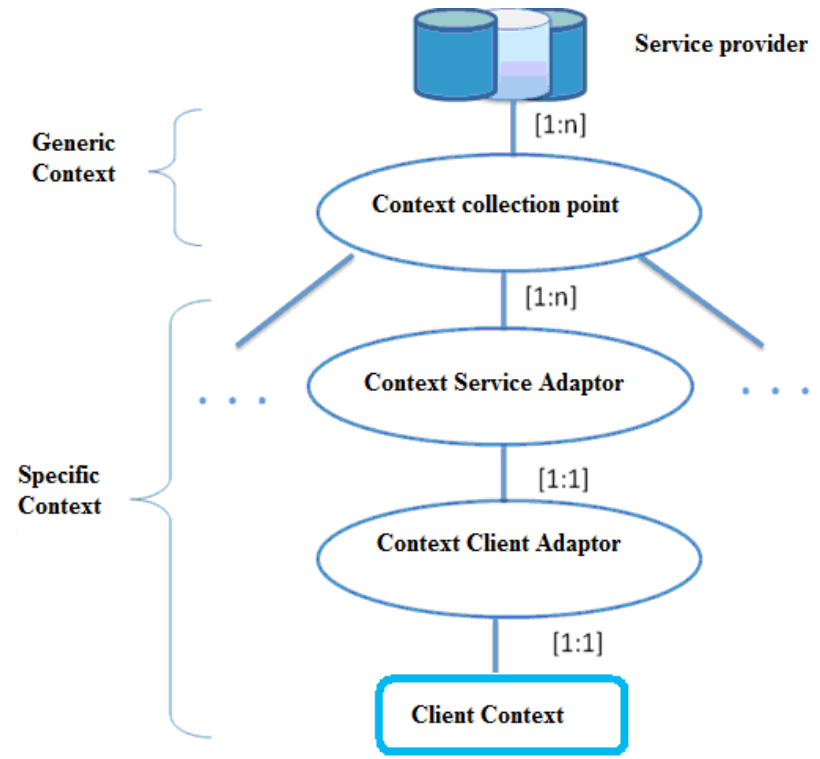

Figure 3. Context management scheme [5].

\section{RELATED WORKS}

\subsection{A Handover Based Context Aware}

\section{Scenario}

In [6], authors present a handover scenario based context aware, and discuss how the context information will be handled by the mobile equipment. The corresponding implementation defines the following entities:

- The point of handover decision, which chosen the access router for the handover.

- The point of context collection within the network, which collects and compiles the relevant contextual information resulting from various sources. These information is then transmitted to the handover making decision point.

Indeed, two architectures can be presented [6]:

- The handover decision point is integrated on the mobile equipment. So, the context collection point collects contextual information from different point of attachment, and sends them to the handover decision point in order to choose the next point of attachment.

- The handover decision point and the collection context point are considered as the same point within the network. The context collection point collects contextual information from different point of attachment. Then, the dynamic mobile equipment context must be sent to the handover decision point.

In the following, we present two existing and independent approaches for handover making decisions, which we combine in our proposed approach.

\subsection{A Static Context Exchange Protocol}

This approach requires following preparation stages [6]:

1- Conceive the context attributes and formats

2- Implement an algorithm for the context performance and the handover making decision.

3- Set up a context collection mechanism that compiles the relevant information in specified format

4- Implement triggers to collect and transfer the contextual information at the adequate moment.

Dynamically, the following stages are necessary:

1- Context data exchange before the handover making decision.

2- If necessary, updates of this information can be sent.

3- The mobile equipment uses the following handover procedure, that is started by a connection loss or with the need to change toward a different point of attachment, consisting of: first, collect the dynamic context of the mobile equipment (list of visible points of attachment, signal power, application) Second, Call upon the algorithm with the supplied data and the dynamic context to make the decision of the most suited handover. Third, apply the handover to the chosen point of attachment.

\subsection{Dynamic Acquisition of the Context Awareness Decision Making Agent}

In this approach, we download a software agent [6] that encapsulates the context information and the data performance diagnostic algorithms. In such a way, we avoid the limits of the static context exchange method. This approach is more flexible than the first one and does not require a standard format of the context. This solution requires a software handover making decision point to support a dynamic installation of the agent. We proceed as follows [6]:

1- We prepare an agent at the context collection point by the means of the associated algorithm and the contextual information that we need in the handover making decision point. This stage is necessary when 
the algorithm or the context changes, as for example when we add a new cell or when the user profile changes.

2- Download of the agent before the link change toward the neighbour cell for the handover making decision point

3- The agent is called during the handover as follows: the mobile equipment dynamic context is provided to this agent at the beginning and it will provide the handover decision by the end.

The agent is called during handover, and uses the dynamic contextual information provided by the mobile equipment (for example the current users services). With regard to the previous approach, only the agent must be downloaded, and not all the list of active points of attachment within the considered area. The agent can be considered as a simple hierarchical decision compiled from the data of essential context.

\section{PROPOSED ALGORITHM: A FLEXIBLE WORK ENVIRONMENT SUPPORTING BOTH STATIC AND DYNAMIC CONTEXT EXCHANGE APPROACHES}

The previously explained approaches are two different solutions: in the first one, the network and the mobile equipment, firstly have to agree on a static format for the contextual information; in the second one, a program ( an agent) which must be downloaded by the mobile equipment from the network to understand the contextual information in order to be able to handling them. The difference is that the solution with the added agent is completely flexible counterpart the data structures and the used formats for the contextual information. But the first solution is however limited to a fixed structure of data, for example a table or a decision tree.

In this working environment, we are going to combine both ideas. So, we consider that the algorithm and the contextual information are separately downloaded in different time intervals. We use an agent which is also capable of exchanging the context if there is a structural change of the contexts information. The stages of agent exchanges are as follows :

1- Prepare an agent at the context collection point using the algorithm and the collected contexts data which we need for the handover making decision point

2- Download a new agent which contains the algorithm and the context exchange protocol for handover making decision point.

The stages of context exchange and handover execution are :

1- When the context changes, we exchange the context data (typically during the addition of a new cell, a neighbour one)
2- The agent is called during the handover as follows: at the beginning : the mobile equipment dynamic context and the exchanged context; at the end : the selected point of attachment

We note that the exchange of agents can be applied in various manners. For example, every time the user profile changes, a new agent is installed. Alternately, the agent cannot be updated unless there are changes in the user behaviour or with network services.

\section{SIMULATION CONCEPTION AND DEVELOPPED SCENARII}

\subsection{Simulated Network Topology}

Here we present a common network topology for all conceived scenarios (cf. figure 4). It consists of:

- Five mobile nodes

- A Wimax base station

- A Wifi access point

At the beginning of the simulation, we suppose that all mobile nodes are connected to the WiMax base station.

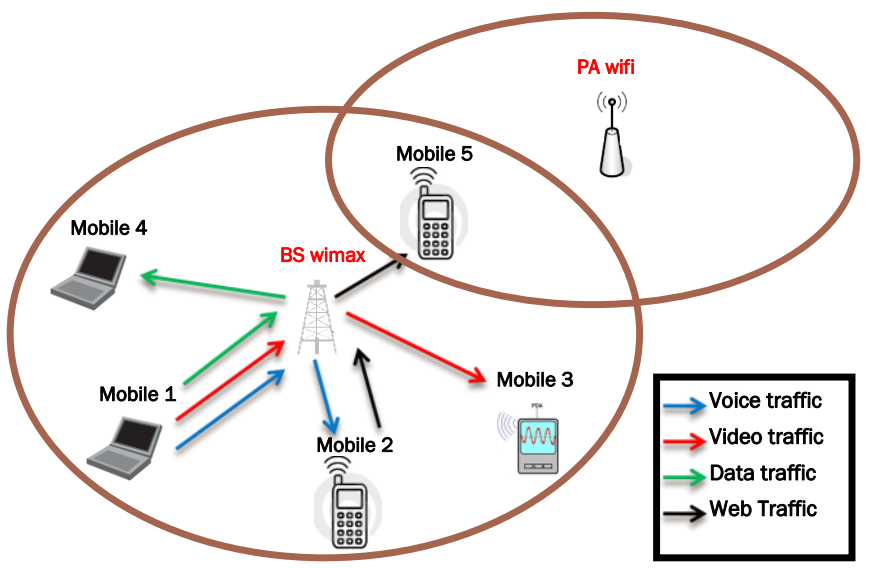

Figure 4. Simulated network topology

\subsection{Traffics Models}

During these simulations, we use the agent SCTP (Stream Control Transmission Protocol) as transport protocol and traffic models as CBR, FTP and Pareto traffic model. Our simulations are done using the network simulator platform. The use of SCTP agent defines mobile node as a core node with multiple interfaces, each one can be connected to a given radio access technology network interface. We can therefore say that the agent SCTP enables the delivery of traffic from multiple interfaces. This option provided by the SCTP agent, is called multi-homing. Our goal is to use multi-homing feature of SCTP to improve transmission performance.

We will simulate voice and video traffics using CBR application protocol modeling, data traffic with FTP protocol and Web traffic with Pareto model. We defined the characteristics of simulated connections, in terms of bit rate and packet size in Table 1. 
Table I. Traffic parameters considered during simulations

\begin{tabular}{|l|l|l|}
\hline Traffic type & Bit Rate & Packet size \\
\hline Voice & $9.6 \mathrm{Kbit} / \mathrm{s}$ & 240 octets \\
\hline Video & $20 \mathrm{Mbit} / \mathrm{s}$ & 1500 octets \\
\hline Data & $100 \mathrm{Kbit} / \mathrm{s}$ & 1460 octets \\
\hline Web & $10 \mathrm{Mbit} / \mathrm{s}$ & 500 octets \\
\hline
\end{tabular}

In the following, we will implement a few scenarios to conceive a handover mechanism based on the context awareness concept.

\subsection{Developed scenarios}

\subsubsection{Common description}

All our simulations are developed with NS2.33[7].We create a WiFi attachment point, a WiMAX base station and five mobile nodes, each having two interfaces, one interface for Wifi access type and another for WiMax connection.

\subsubsection{First scenario}

In this section, we will describe a scenario example that can be encountered in a mobile radio network. This scenario will include the handover mechanism based on a context awareness concept.

First, the mobile "node 1" requests three types of traffic connections simultaneously :

- A voice traffic is triggered with the mobile node "node 2"

- A video traffic is triggered toward the mobile node "node 3"

- A data traffic is triggered toward the mobile node "node 4" These different connections types have the features defined in Table 1.

The mobile node "node 1" activates these various traffic through its WiMax interface via WiMax base station "WiMax BS" to the corresponding mobile receiver node WiMax interfaces.At different times, the mobile "node 1" began to send different traffic flows that we have described above. Activated traffic applications, as shown in figure 5, pass through the WiMax base station "BS wimax" which, in turn, forwards them to their corresponding destinations.

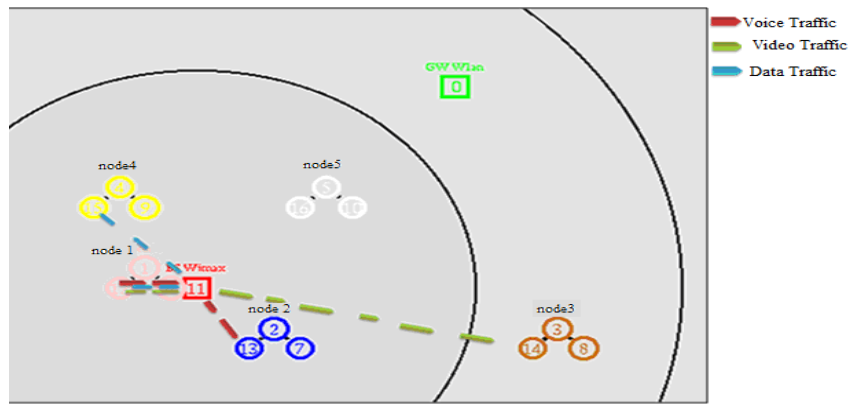

Figure 5. Scenario 1: Traffic flows sent by node1
At the same time, the mobile node "node 1" moves toward the WiFi access point "GW wlan". During its movement, "node1" keeps the different mobile communications with the correspondent receivers. To improve the connection quality, a handover is triggered based on the concept of context awareness, where the signal strength received from WiMax base station "BS wimax" falls below the received signal power from the Wifi point of Attachment "GW wlan". Indeed, since we used the TwoRayGround propagation model, signal power is influenced by the distance (not by obstacles, noise,...). The mathematical expression of the received power is given by :

$\mathrm{P}_{\mathrm{r}}=\mathrm{P}_{\mathrm{t}} * \mathrm{G}_{\mathrm{r}} * \mathrm{G}_{\mathrm{t}} *\left(1 /(4 * \pi *(\mathbf{d} / \boldsymbol{\lambda}))^{2}\right.$

Where : $\mathbf{P}_{\mathbf{t}}$ : is the transmitted signal power; $\mathbf{G}_{\mathbf{r}}$ : the receiver antenna gain; $\mathbf{G}_{\mathbf{t}}$ the transmitter antenna gain $; \mathbf{d}$ : the distance separating mobile node to the considered serving network node and $\square$ : is the system wave length that depends on the system functional frequency.

Switching to the "GW wlan" is based on specific criteria that define a smart handover. In our implementation we consider the throughput of various connections. To reduce the load of "BS wimax", we redirected the video traffic, which is the most demanding in terms of throughput, to the "GW wlan" so the video traffic exchange between mobile node "node 1" and mobile node "node 3" is ensured through Wifi interfaces with the "GW wlan" as shown in figure 6.

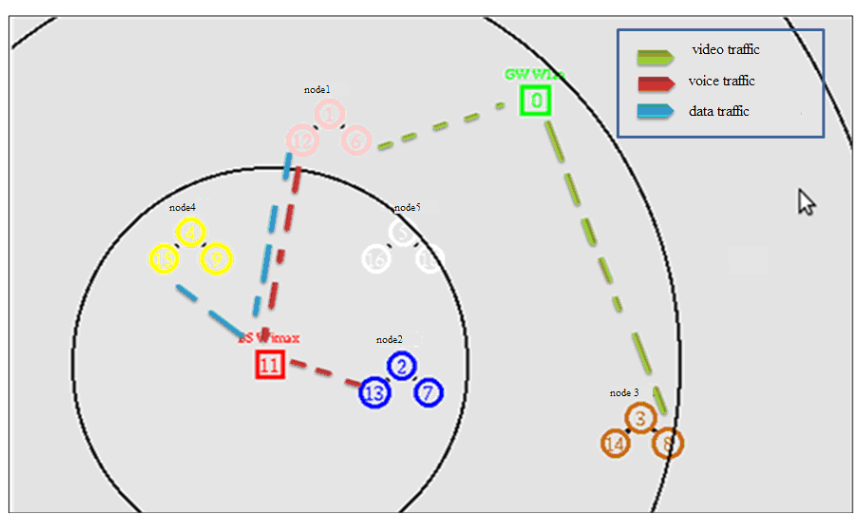

Figure 6. First scenario handover based context awareness execution

Now, we will focus on the different variations of signal power throughout the mobile "node 1" movement. Referring to the obtained simulation results, we observe at the beginning of the simulation, the signal strength received from the WiMax BS is greater than that received from the Wifi point of attachment (cf. figure 7). 


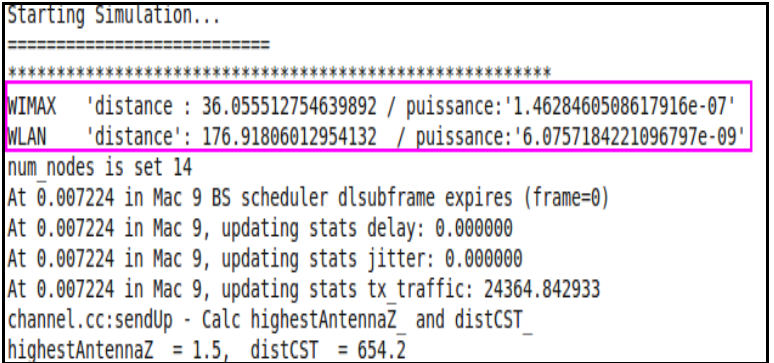

\section{Figure 7. First stage in the Scenario 1 simulation}

During movement of the mobile transmitter "node 1", the received powers from the WiMAX BS and from the attachment point Wifi, progressively approach, as outlined in the figure 8 .

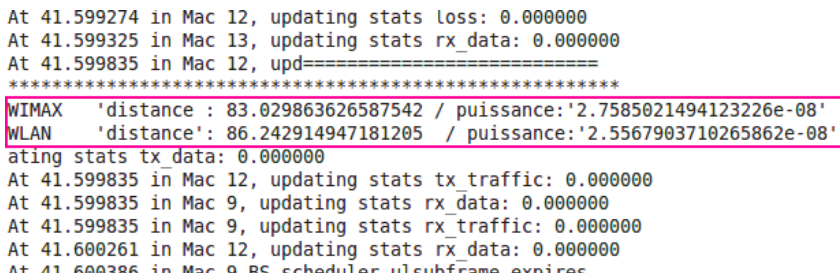

\section{Figure 8. Scenario 1 intermediate simulation stage (before handover}

When the received power from Wifi point of attachment becomes higher than the received signal power level from the WiMAX base station, the handover is triggered by redirecting video traffic to the Wifi attachment point which in turn transmits it to the "Node 3" (cf. figure 9).

At 41.699274 in Mac 10, updating stats loss: 0.000000 $8460000.001 *$ At 41.699274 in Mac 12, updating stats rx traffic: 8460000

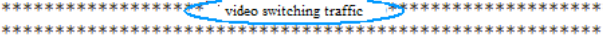
WIMAX 'distance : 87.855453400566944 / puissance: '2.4637947596212365e-08' WLAN 'distance': 83.191476814936209 / puissance: '2.7477948675037275e- $\theta$ '

At 41.699274 in Mac 12, updating stats loss: 0.000000 At 41.699325 in Mac 13, updating stats rx data: 0.000000 At 41.700386 in Mac 9 BS scheduler ulsubframe expires

\section{Figure 9. Scenario 1, handover execution illustration}

\subsubsection{Second scenario}

In this scenario, we added web traffic to be delivered by the mobile "node 2 " to the mobile receiver node "node 5 ". We have identified some features for web traffic, which corresponds to a Pareto model, described in Table 1. Initially, we are interested in mobile "node 2 " transaction, which will send web traffic to "node 5" throughout during its movement. So we will follow the various movements of the mobile transmitter and the different directions of web traffic in order to perceive the radio link switching steps.

We note that web traffic goes through the WiMax base station "WiMAX BS." This action is due to the fact that the received signal power from WiMax base station is greater than the received signal power from Wifi point of attachment. The mobile "node 2" moves approaching slowly the WiFi attachment point. During mobile node "node 2" movement, we compare the received power from WiMax base station to the received one from WiFi attachment point. When the received power from $\mathrm{WiFi}$ attachment point exceeds the one from WiMax BS, without significant quality degradation which means that the received power from the WiMax BS is still greater than a minimum required quality threshold value, we execute the handover.

The handover execution means that we force the mobile "node 2" to issue web traffic using its Wifi interface and the mobile receiver "node 5 " to receive this traffic by its Wifi interface using the following pseudo code. Such behavior is possible with the multihoming feature of SCTP protocol.

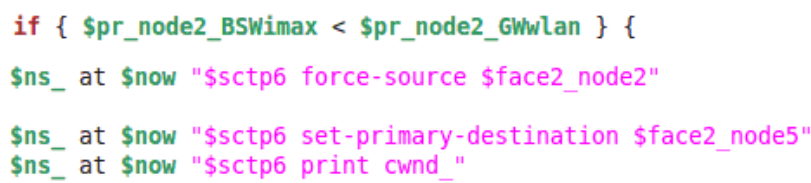

Now, after viewing the web traffic switching radio link based on the received power calculation, we can check the switching respected conditions with the simulation event tracking. The figure 10 shows the received power values from the WiMAX BS and from the Wifi point of attachment at the beginning of the simulation

using backward compatible Agent/CBR; use Application/Traffic/CBR instead using backward compatible Agent/CBR; use Application/Traffic/CBR instead Starting Simulation. .

******************* Pour noeud 2 (trafic web) $* * * * * * * * * * * * * * * * * * * * * * * * * * * * * * * * 4$ WIMAX 'distance : 36.055512754639892/puissance: '1. 4628460508617916e-07' WLAN 'distance': 138.92443989449805 / puissance: '9.8533671819706194e-09'

Figure 10. $\quad$ First stage in the Scenario 2 simulation

During mobile "Node2" movement, we note that the distance between the mobile transmitter "Node 2 " and the WiMAX base station increases and the distance between the mobile "Node2" and WiFi attachment point decreases (cf. figure 11).

At 45.695581 in Mac 11, updating stats de $\bar{l}$ ay: 0.000021

******************* Pour noeud 2 (trafic web) $* * * * * * * * * * * * * * * * * * * * * * * * * * * * * * *$ WIMAX 'distance : 80.342649850663776 / puissance: '2.9461148485898481e-08' WLAN 'distance': 81.374927885838048 , puissance: '2.8718433214542615e-08' 45.695581 in Mac 11, updating stats jitter: 0.000021 At 45.695581 in Mac 11, updating stats tx_traffic: 11920909.091611

Figure 11.

Scenario 2 intermediate simulation stage (before handover)

The received power from WiMax BS decreases gradually until it becomes less than the received power from Wifi attachment point (cf. figure 12) .

At 45.799581 in Mac 11, updating stats jitter: 0.000021 At 45.799581 in Mac 11, updating stats tx_traffic: 11920909.091611 ********************* basculement web $* * * * * * * * * * * * * * * * * * * * * * * * * * * * * * * * * * * * *$ ******************) Pour noeud 2 (trafic web) $* * * * * * * * * * * * * * * * * * * * * * * * * * * * * * * *$ WIMAX 'distance : 84.971563377337802 / puissance: '2.6338725627930865e-08' WLAN 'distance': 77.340416421925724 i puissance: '3. 1792813593229294e-08

Figure 12.

Scenario 2, handover execution beginning illustration.

Here the web traffic switching is triggered. 


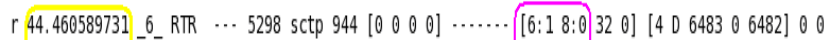

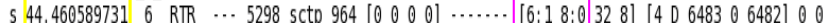

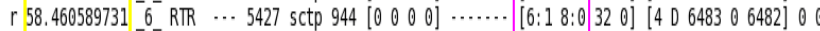
s $58.460589731\left[\begin{array}{l}6 \\ 6\end{array}\right.$

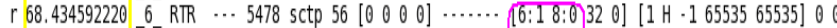

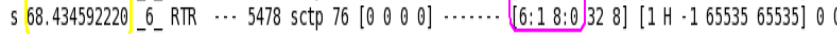

Figure 18.

Scenario 1: detailed simulation events after Handover success.

Here we calculate the handover latency time:

Handover Latency $=$ time of the first event after executing the handover - time of the last event before the handover;

based on simulation results we obtain :

$$
\text { Handover Latency }=44.460589731-38.580267750=
$$

$$
5.880321981 \mathrm{sec}
$$

This latency is large enough to switch the video traffic; it is also the major drawback of the handover. If we use the TCP transport protocol, the latency will be translated by a connection failure. While for our implementation we use the SCTP protocol that keeps the connection during this latency time, but we will have no transmitted traffic during a time interval about $5 \mathrm{sec}$.

At this stage, we visualize the number of lost packets variations throughout the simulation duration for video exchanged traffic between "node 1" and "Node 3". This observation is made in two cases; the first case without executing the handover and the second case with implementation of the handover based context awareness as shown in Figure 19.

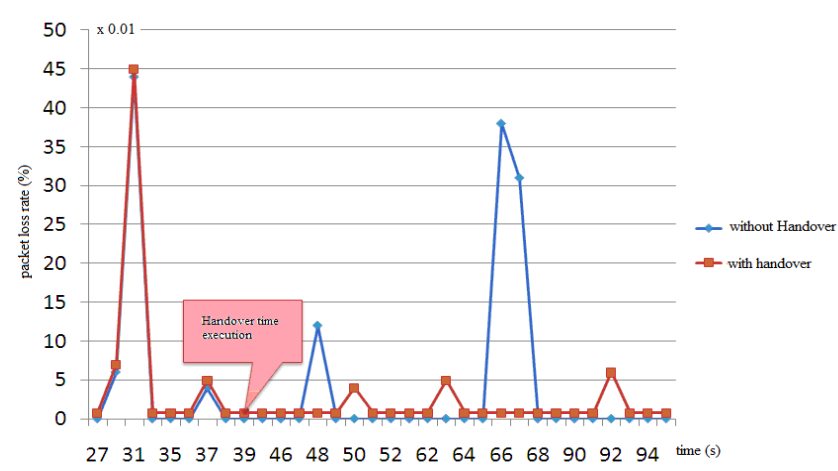

Figure 19. Scenario 1: packet loss rate variation without and with handover execution

The instant ' $41 \mathrm{~s}$ ' represents the execution time of handover for the second case. For this reason we will compare the rate of packet loss before and after this moment. Before $\mathrm{t}=41 \mathrm{~s}$ : we notice that in both cases we have the same packet loss rate, since the handover has not yet triggered.

After $t=41 \mathrm{~s}$ : the number of packets lost in the first case, without handover execution, is more important than the second one (with handover execution), this is insured by link switching with handover execution. Indeed, the packet loss rate is $0.1 \%$ for the first case, while it is about $0.06 \%$ in the second case performing the handover. We note that the loss rate decreases when we execute the handover based context awareness. In fact, the handover based context awareness is triggered not when the radio link quality is decreased but is triggered even we have a certain guaranteed quality of service in order to be better served. This result justifies the use of intelligent handover that improves the connection quality by reducing the packet loss rate.

\subsection{Second Scenario}

By examining the trace file, we can verify that the handover execution is successful. Indeed, web traffic, just before the radio link switching, is transmitted through the mobile node "node 2" WiMax interface, numbered '13 ', and the receiving node "node 5" receives via its WiMax interface, numbered '16'. This traffic passes through the WiMax base station indexed ' 11 ' as is illustrated by figure 20 .

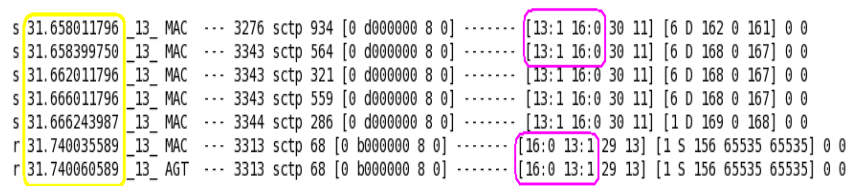

Figure 20. Scenario 1: detailed simulation events before Handover

After executing the handover, web traffic switches from WiMax interface to Wifi one. The "Node 2" sends the web traffic via its Wifi interface with the index '7', the mobile receiver "node 5 " receives through its Wifi interface with the index '10' (cf. figure21).

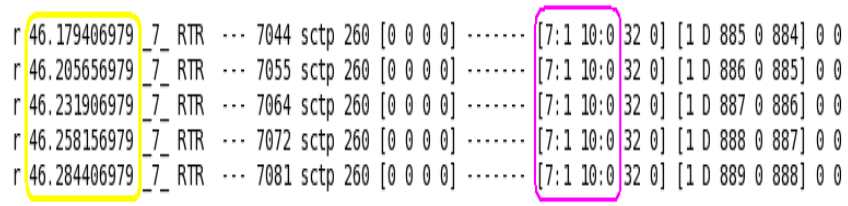

Figure 21.

Scenario 2: detailed simulation events after Handover success.

Let's calculate the generated latency for the handover execution, according to equation (2), so we obtain:

Handover latency $=46.179406979-31.740060589=$ $14.43934639 \mathrm{~s}$

We note that this latency is large enough to switch the web service, this may cause a connection drop which may be a disadvantage of the handover based context awareness.

To address the effectiveness of our simulation, we use an evaluation of the simulated scenario in terms of packet loss rate. We will compare the packet loss rate without and with the execution of handover to assimilate the advantage of the performance of handover as shown in Figure 22. 


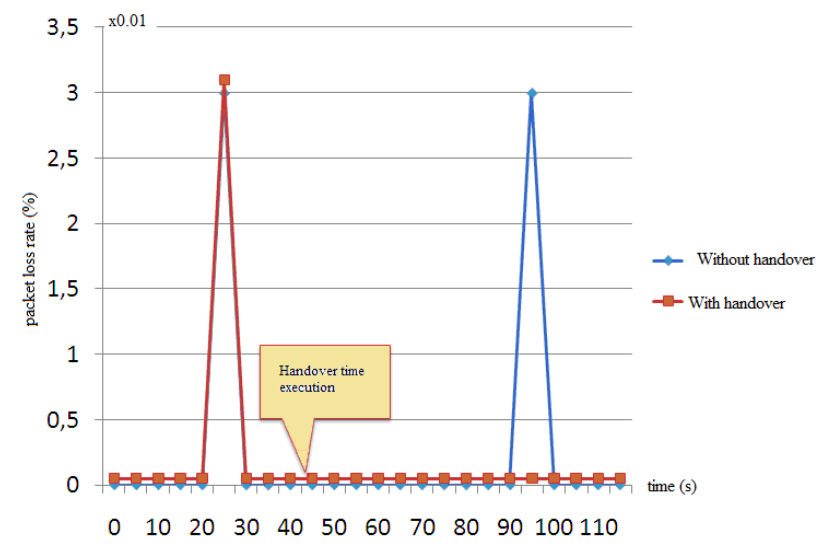

Figure 22. Scenario 2: packet loss rate variation without and with handover execution

These results clearly explain the use of intelligent handover to improve the end to end connection. In fact, we note that during the handover execution stage, precisely at $\mathrm{t}=45$ second, we will have no more packet loss. Indeed, the packet loss rate is equal to $0.0048 \%$ for the first case (without handover, data transmission continues over WiMax radio interface), while it is about $0.0026 \%$ for the second case corresponding to the case with a handover based context awareness execution. Always the same finding: the packet loss rate decreases when we execute the handover based context awareness. This result justifies that the use of intelligent handover improves the connection by reducing the packet loss rate.

\subsection{Third Scenario}

By analyzing the trace file, we can verify the success of our proposed handover based context awareness proposed scheme. Before execution of the handover, video traffic is transferred through the transmitting mobile node "node 1" WiMax , numbered ' 12 ', and the receiving mobile node "node 3 " receives this traffic via its WiMax interface, indexed '14', as shown by figure 23 .

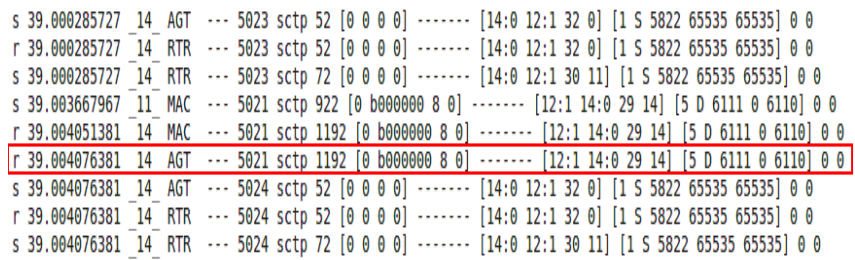

\section{Figure 23. Scenario 3: detailed simulation events} before video traffic radio link switching

For data traffic, it is transmitted firstly through mobile node "node 1" WiMax , numbered '12', and the receiving mobile node "node 4" receives this traffic via its WiMax interface, indexed ' 15 ', as shown by figure 24 .

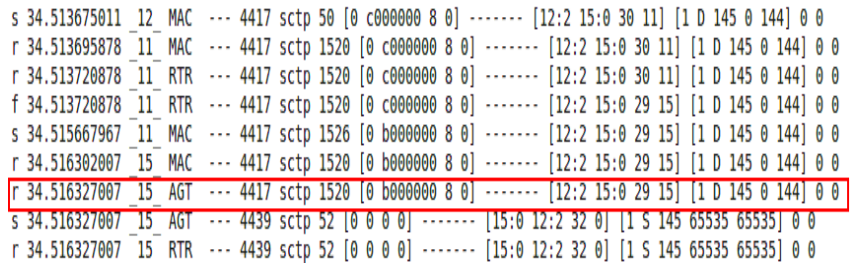

Figure 24. Scenario 3: detailed simulation events before data traffic radio link switching

After executing the handover, the video traffic switch interfaces. The "Node 1" sends the video traffic across its Wlan interface, index '6', and' Node 3 'receives this traffic flow through its Wlan interface indexed ' 8 ' as illustrated by figure 25.

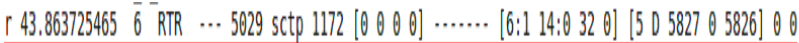

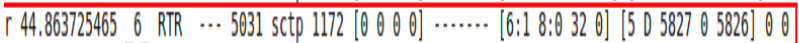

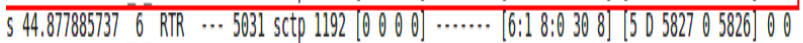

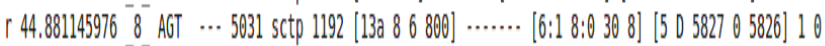

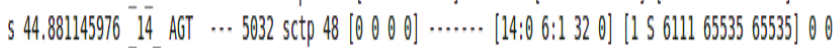

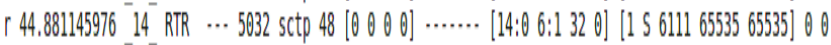

Figure 25. Scenario 3: detailed simulation events after video traffic radio link switching

For data service, the transmitter mobile node "Node 1" sends the data traffic flow after executing handover based context awareness over its Wlan interface indexed ' 6 'to the mobile node "node 4" Wlan interface indexed ' 9 ', as can be seen in figure 26 .

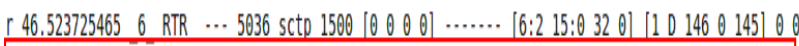

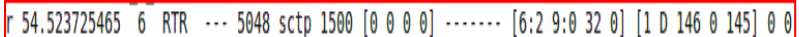

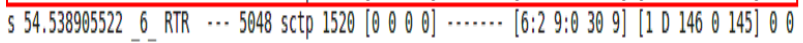

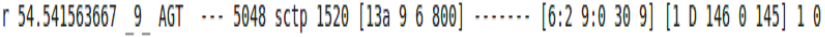

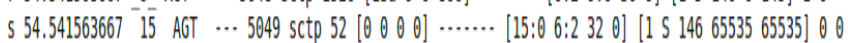

Figure 26. Scenario 3: detailed simulation events after data traffic radio link switching

Now let's calculate the required latency required for the handover execution related to video and data services:

For the video service, based on equation 2 :

Video HO_Latency $=44.863725465$ $39.004076381=5.859649084$ seconds

For data service, using the formula given by equation 2 :

Data HO_Latency $=54.523725465$ $34.516327007=20.007398458$ seconds

From these results, we find that the latency data service is more important than the latency of video service since the video traffic requires more bandwidth than the data traffic, so it will be served firstly. Otherwise, since we used the SCTP transport protocol, the latency generates an instantaneous 
switching packet transfer, not a connection failure, such is with TCP case.

Here we will conduct an evaluation of the obtained results to assess the importance of implementing the handover based context awareness in specific cases. Figure 27 we can note the effect of the handover based context awareness execution on packet loss rate.

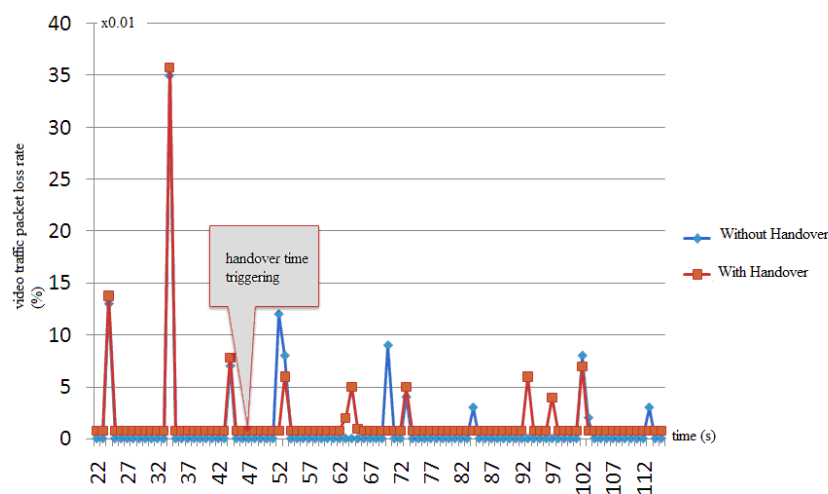

Figure 27. Scenario 3: video trafficpacket loss rate variation without and with handover execution

We note that there is no packet loss at the time of the execution of the handover, which acts effectively on the connection quality. Indeed, the packet loss rate calculated in the scenario without the implementation of the handover is equal to $0.21 \%$, while it reaches $0.18 \%$ in the second case, with handover implementation. So, we check that the packet loss rate is higher in the first case than in the second, which again verifies the effectiveness of the handover based context awareness on the end to end connection quality.

\section{CONCLUSION}

In this paper, we simulated three scenarios to highlight the performance of the handover based on the concept of context awareness. This concept supposes that we can trigger a radio link switching without waiting link quality degradation, so we can configure our radio connections dependant on radio access technologies availability. During the simulations, we have identified the importance of the handover to improve the end to end connection quality by reducing the packet loss considering different traffic flows configurations, which comprise single service or simultaneous services. We note that the handover based context awareness provides an intelligent approach for mobility management mechanisms and presents an effective solution to increase network performance, but the generated handover latency is important and needs to be enhanced by proposing other improvements for such a concept which constitutes our interest for future publications.

\section{REFERENCES}

[1] Anind K. Dey and Gregory D. Abowd, Towards a Better Understanding of Context and Context-Awareness, HUC '99 Proceedings of the 1st international symposium on Handheld and Ubiquitous Computing, 1999.

[2] Ouahiba FOUIAL. "Découverte et fourniture des services applicatifs dans les environnements mobiles". Thesis report Telecom-Paris. 2004.

[3] Ricardo Matos and Susana Sargento, Context-Aware Connectivity and Mobility in Wireless Mesh Networks, Lecture Notes of the Institute for Computer Sciences, Social Informatics and Telecommunications Engineering, Volume 32 Part 2 pp- 49-56, 2010.

[4] Thomas Hofer, Wieland Schwinger, Mario Pichler, Gerhard Leonhartsberger, Josef Altmann and Werner Retschitzegger, Context-Awareness on Mobile Devices the Hydrogen Approach, Proceedings of the 36th Annual Hawaii International Conference on System Sciences (HICSS'03), Track 9 Volume 9, 2003.

[5] Paulo Mendes, Chiristian Prehofer, Qing Wei. "Context Management with programmable mobile networks". 2003. pp. 3-5

[6] Christian Prehofer, Nima Nafisi, Qing Wei. "A framework for Context-Aware handover Decision". 2003. pp. 7-10

[7] Network Simulator

NS2.33 http://www.isi.edu/nsnam/ns. 